\title{
Single venom-based immunotherapy effectively protects patients with double positive tests to honey bee and Vespula venom
}

\author{
Johanna Stoevesandt ${ }^{1 *}$, Bernd Hofmann ${ }^{1,2}$, Johannes Hain ${ }^{3}$, Andreas Kerstan ${ }^{1}$ and Axel Trautmann ${ }^{1}$
}

\begin{abstract}
Background: Referring to individuals with reactivity to honey bee and Vespula venom in diagnostic tests, the umbrella terms "double sensitization" or "double positivity" cover patients with true clinical double allergy and those allergic to a single venom with asymptomatic sensitization to the other. There is no international consensus on whether immunotherapy regimens should generally include both venoms in double sensitized patients.

Objective: We investigated the long-term outcome of single venom-based immunotherapy with regard to potential risk factors for treatment failure and specifically compared the risk of relapse in mono sensitized and double sensitized patients.

Methods: Re-sting data were obtained from 635 patients who had completed at least 3 years of immunotherapy between 1988 and 2008. The adequate venom for immunotherapy was selected using an algorithm based on clinical details and the results of diagnostic tests.

Results: Of 635 patients, 351 (55.3\%) were double sensitized to both venoms. The overall re-exposure rate to Hymenoptera stings during and after immunotherapy was 62.4\%; the relapse rate was $7.1 \%$ (6.0\% in mono sensitized, $7.8 \%$ in double sensitized patients). Recurring anaphylaxis was statistically less severe than the index sting reaction $(P=0.004)$. Double sensitization was not significantly related to relapsing anaphylaxis $(P=0.56)$, but there was a tendency towards an increased risk of relapse in a subgroup of patients with equal reactivity to both venoms in diagnostic tests $(P=0.15)$.

Conclusions: Single venom-based immunotherapy over 3 to 5 years effectively and long-lastingly protects the vast majority of both mono sensitized and double sensitized Hymenoptera venom allergic patients. Double venom immunotherapy is indicated in clinically double allergic patients reporting systemic reactions to stings of both Hymenoptera and in those with equal reactivity to both venoms in diagnostic tests who have not reliably identified the culprit stinging insect.
\end{abstract}

Keywords: Anaphylaxis, Double sensitization, Field sting, Honey bee, Hymenoptera venom, Immunotherapy, Relapse, Risk factor, Treatment failure, Vespula

\footnotetext{
* Correspondence: Stoevesandt_J@klinik.uni-wuerzburg.de

'Department of Dermatology and Allergy, University Hospital Würzburg,

Würzburg, Germany

Full list of author information is available at the end of the article
} 


\section{Background}

Implementation of venom immunotherapy (VIT) in a Hymenoptera venom allergic patient involves a number of individual decisions weighing aspects of treatment efficacy and optimal protection versus costs, feasibility, and patient convenience. International guidelines [1-3] provide elaborate instructions on dosing and duration of VIT, but there is no definite statement on which venoms to include for immunotherapy if sensitization to more than one venom is detectable.

Sensitization to both honey bee and Vespula venom is a common finding in up to 59\% [4] of Northern European Hymenoptera venom allergic patients. The terms "double sensitization" or "double positivity", however, refer to the results of diagnostic tests rather than to their clinical significance. In the past decades, substantial efforts have been made to differentiate between venom "cross-reactivity" due to partial sequence identity of protein allergens or cross-reactive carbohydrate determinants and "independent double sensitization" by means of serological [4-11] or cellular tests [12-14]. Recently, assays of species specific IgE to recombinant major allergens such as rApi $\mathrm{m} 1$ and rVes v5 and lately rVes v1 [4-6] became commercially available for diagnostic testing. "Independent" or major allergen-based double sensitization has been considered an indication for double VIT $[4,6,10,13]$, and some experts recommend that VIT should be extended to all venoms for which test results are positive and patients might potentially react to [15]. Still, there is a lack of clinical studies investigating whether double sensitization - regardless of its pattern or origin - is objectively associated with an increased risk of future anaphylactic reactions to both Hymenoptera venoms and therefore necessitates double VIT, entailing a significant increase of health care costs and time and effort.

Our study retrospectively examines a large cohort of patients who were treated by single venom-based immunotherapy at a standard dose of $100 \mu \mathrm{g}$ per injection for at least 3 years between 1988 and 2008. It was designed to evaluate the risk of relapsing Hymenopterasting induced allergic reactions during maintenance VIT and after its discontinuation, to analyze risk factors for treatment failure, and to specifically compare the risk of field sting-induced anaphylaxis in mono sensitized and double sensitized patients.

\section{Methods}

\section{Patients}

In this retrospective single centre observational cohort study covering a period of 20 years (1988 to 2008), 721 patients were screened for study inclusion criteria. Patients with proven honey bee or Vespula venom allergy were eligible for evaluation if single venom-based immunotherapy had been performed according to international guidelines [1-3] and discontinued after a minimum period of 3 years. Patients who had received VIT with both venoms, those who stopped treatment before 3 years duration, and those who did not present for follow-up visits were excluded from the study. All patient-related procedures were part of routine diagnostic practice; written informed consent was obtained for allergologic work-up and initiation of VIT.

\section{Collection of data}

Patients fulfilling study inclusion criteria were identified from patient files. A detailed follow-up history using a standardized questionnaire was routinely taken on the occasion of control visits in our allergy clinic. Data collection included information on patient-specific data (age at the time of VIT initiation, sex, underlying atopy or asthma) and on Hymenoptera field stings before, during and after VIT (date, number of stings, culprit insect, symptoms and severity of anaphylaxis, selfadministration of medication, emergency treatment). Results from allergy testing (intradermal skin test results, specific IgE, baseline serum tryptase) and information on the course of VIT (time-interval between index-sting and VIT initiation, date of VIT initiation and duration of VIT) were retrieved from the patients' files. Patients reporting relapses after discontinuation of VIT were invited for retesting and advised to restart VIT.

\section{Grading of anaphylaxis}

Severity of anaphylaxis was classified as mild, moderate or severe with respect to cutaneous, respiratory, cardiovascular, gastrointestinal, and neurological symptoms according to a modified version of the system proposed by Muraro [16] as has been described previously [17].

\section{Allergy testing}

Intradermal and serological tests were performed before initiation and prior to cessation of VIT. Intradermal skin tests with 10-fold serial dilutions of honey bee and Vespula venom (ALK-Abelló, Wedel, Germany) were carried out according to international guidelines $[18,19]$. Intradermal skin test responses at endpoint concentrations of less than or equal to $1.0 \mu \mathrm{g} / \mathrm{mL}$ were considered "positive" when resulting in a minimum wheal of $5 \mathrm{~mm}$. Venom-specific IgE levels were determined using an enzyme immunoassay technique (DPC Biermann, Bad Nauheim, Germany) before 1998, which was replaced by the ImmunoCAP ${ }^{\mathrm{m}}$ method (Thermo Fisher Scientific, Schwerte, Germany). The results were categorized by semi-quantitative classes ranging from 0 to 6 . Classes higher than or equal to 1 were considered "positive". Additional diagnostic methods including determination of specific IgE to recombinant major venom allergens 


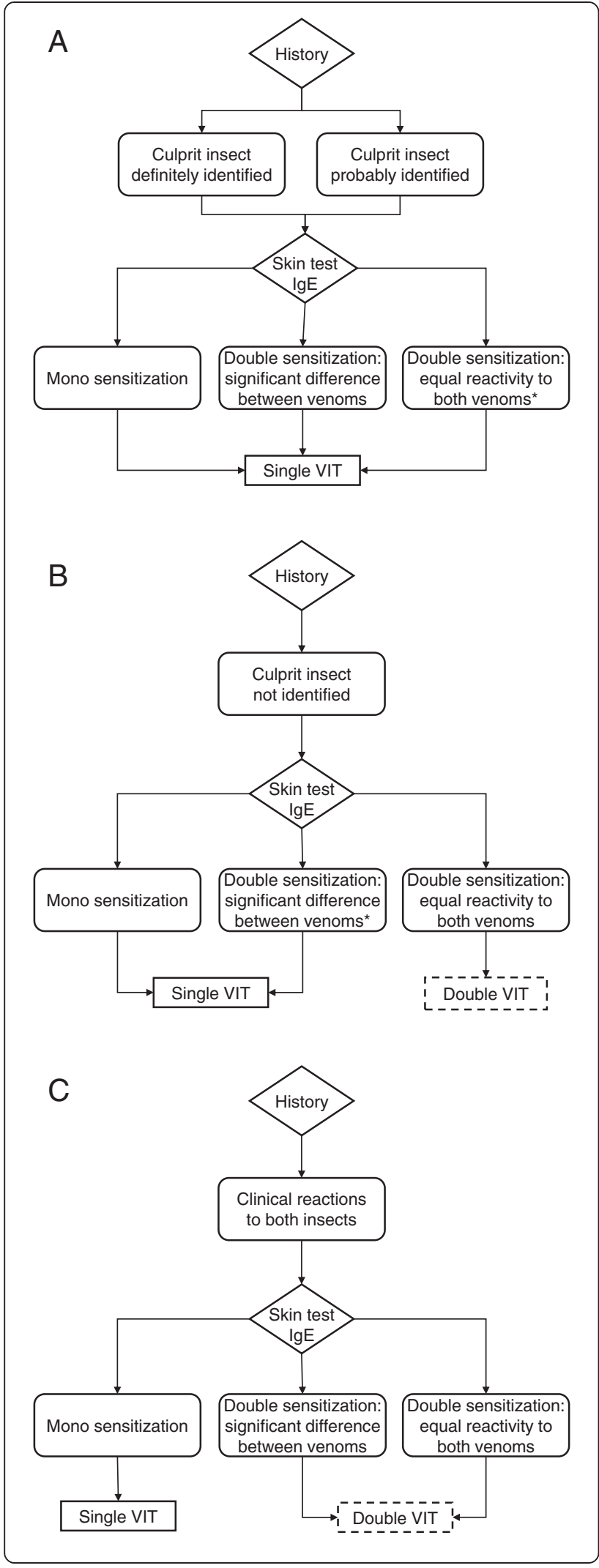

Figure 1 Algorithm for the selection of venom for

immunotherapy. A. Reliable visual identification of the culprit stinging insect or sufficiently certain identification based on additional sting-related information (patient's location and activity, time of the year, presence of a stinger left in the skin): administer single VIT. B. Culprit insect not identified: administer single VIT in mono sensitized patients or if sensitization to a single venom is significantly stronger than to the other (i.e. at least one serological class and one concentration step in intradermal tests). Administer double VIT in patients with equal reactivity to both venoms. C.

Patients reporting anaphylaxis to stings of both insects: administer single VIT in mono sensitized patients. Administer double VIT in all patients with double sensitization. ${ }^{*}$ Consider double VIT if additional risk factors are present (e.g. comorbidities, severe anaphylaxis to index sting, high degree of exposure). Dashed boxes: patients receiving double VIT were not eligible for study inclusion.

were not routinely available during the study period. Determination of the baseline serum tryptase was introduced in routine diagnostic practice in 2002 and was performed using ImmunoCAP Tryptase ${ }^{\mathrm{m}}$ (Thermo Fisher Scientific) in a subgroup of patients with a history of severe stinginduced anaphylaxis. Tryptase levels exceeding $11.4 \mu \mathrm{g} / \mathrm{L}$ $\left(95^{\text {th }}\right.$ percentile of the general population) were considered as elevated.

\section{Choice of venom for VIT}

The venom for immunotherapy was selected based on a detailed clinical history and the results of skin and serological tests, taking into account the respective degree of reactivity to both venoms (Figure 1).

\section{VIT venoms and protocols}

VIT was administered according to international guidelines [1-3] using standardized allergen extracts containing Vespula or honey bee venom. Reless ${ }^{\mathrm{Tm}}$ (ALK-Abelló, Wedel, Germany) was used from 1988 to 1999, and ALKlyophilisiert SQ $\mathrm{SQ}^{\mathrm{mm}}$ and ALK-depot SQ ${ }^{\mathrm{m}}$ (ALK-Abelló, Wedel, Germany) were used for build-up and maintenance phase since 2000. Before 2007, initiation of VIT was performed using a 5-day rush protocol which was subsequently replaced by a 3-day rush protocol. The maintenance dose of $100 \mu \mathrm{g}$ was administered every 4 to 8 weeks according to the manufacturer's instructions. Treatment was discontinued within 3 to 5 years in the majority of cases (96.5\%); 22 patients (3.5\%) pursued extended VIT for individual reasons.

\section{Definition of patient groups for statistical analysis}

Patients were defined as "double sensitized" in the case of a positive intradermal skin test response and/or serological detection of specific IgE to both, honey bee and Vespula venom. A subgroup of 116 individuals comprised patients with equal reactivity to both venoms in diagnostic tests as well as a small number of patients $(n=14)$ with discrepant diagnostic results (i.e. skin test 
reactivity was stronger to one and serum specific IgE to the other venom).

\section{Statistical analysis}

Results are presented as numbers and frequencies for categorially scaled measures or as median and range for metrically scaled data. In case of metric data, the Wilcoxon signed-rank test was performed for the comparison of two dependent measures and the Mann-Whitney test for unpaired samples. To assess the relationship between two independent categorial measures, the chi-square test or the exact test of Fisher were used as appropriate. In case of dependent categorial data the Bower test was conducted. The binomial test was done for the comparison of two proportions. All tests were two-tailed and differences were considered statistically significant if $P<0.05$. The software package used was IBM SPSS for Windows, version 19.0.

\section{Results}

\section{Clinical data}

The files of 721 patients with proven honey bee or Vespula venom allergy who had received VIT between 1988 and 2008 were screened for study inclusion criteria. Of 679 individuals who had completed at least 3 years of VIT, 14 patients who had received VIT with both venoms were excluded from evaluation. 30 patients were lost to follow-up, e.g. due to change of residence or death from causes unrelated to Hymenoptera venom allergy. Thus, complete data of 635 patients who had received single venom-based immunotherapy were available for evaluation as summarized in Table 1. The median age at the time of VIT initiation was 39 years (ranging from 5 to 77 ). The sex

\section{Table 1 Clinical data of the cohort}

\begin{tabular}{lcc}
\hline & $\mathbf{n}$ & $\%^{*}$ \\
\hline Number of patients & 635 & \\
Median age at time of VIT-initiation (years) (range) & 39 & $(5-77)$ \\
Male/female & $322 / 313$ & \\
Results of allergy testing & & \\
$\quad$ Vespula (vulgaris/germanica) mono sensitization & 251 & 39.5 \\
$\quad$ Honey bee (Apis mellifera) mono sensitization & 33 & 5.2 \\
$\quad$ Double sensitization & 351 & 55.3 \\
Severity of anaphylaxis to pre-VIT index field sting & & \\
$\quad$ Large local reaction & 8 & 1.3 \\
$\quad$ Grade I (mild) & 171 & 26.9 \\
$\quad$ Grade II (moderate) & 321 & 50.6 \\
$\quad$ Grade III (severe) & 135 & 21.3 \\
Treatment course & & \\
$\quad$ Mean duration of VIT (years) (range) & 3.9 & $(3-13)$ \\
$\quad$ Mean observation time after VIT (years) (range) & 7.0 & $(0-17)$ \\
\hline * Percentages refer to the total study group of 635 patients. &
\end{tabular}

* Percentages refer to the total study group of 635 patients. ratio was almost balanced with only a slight male predominance (50.7\%). Of 635 venom allergic patients, 351 (55.3\%) were double sensitized to honey bee and Vespula venom as detected by intradermal tests or serum specific IgE. The majority of double sensitive cases $(n=160)$ were detected by both, serologic and intradermal tests (Table 2). 107 patients were double positive only serologically, and 84 only in skin tests. Double sensitization was more prevalent in individuals with honey bee venom allergy compared to those who had reacted to Vespula stings $(77.9 \%$ versus 48.4\%, P < 0.001). 135 (21.3\%) patients had a history of severe (grade III) reactions following pre-VIT Hymenoptera field stings, $321(50.6 \%)$ index sting reactions were moderate (grade II), and $171(26.9 \%)$ were classified as mild (grade I). In 8 venom-sensitized patients $(1.3 \%)$ with a history of large local sting reactions, VIT had been performed for individual reasons (mainly loss of quality of life due to fear of Hymenoptera stings). The mean duration of VIT was 3.9 years (ranging from 3 to 13) years. In the whole study population, no major side effects of VIT injections were reported. The mean observation time following discontinuation of VIT was 7.0 years (ranging from 1 month to 17 years).

\section{Skin test and serological reactivity before and by the end of VIT}

Skin test reactivity and specific IgE significantly decreased during VIT $(P<0.001)$. By the end of treatment, intradermal tests with the venom used for immunotherapy were negative in $67(10.6 \%)$ patients, and serum venom-specific IgE was negative $(<0.35 \mathrm{kU} / \mathrm{L})$ in 128 (20.2\%) patients.

\section{Risk of relapse and risk of severe reactions during and after VIT}

396 of 635 patients reported at least one Hymenoptera sting after initiation of VIT by either honey bee $(\mathrm{n}=74)$, Vespula $(\mathrm{n}=233)$, European hornet $(\mathrm{n}=2)$, bumble bee $(\mathrm{n}=1)$ or by an unidentified stinging insect $(\mathrm{n}=86)$, resulting in a re-sting rate of $62.4 \% .174(27.4 \%)$ patients got stung on several occasions: $98(15.4 \%)$ patients had 2 stings, 34 (5.4\%) patients had 3 stings, 20 (3.1\%) had 4 stings, $8(1.3 \%)$ had 5 stings, and $14(2.2 \%)$ had more than 5 stings - several stings on the same event were counted as one sting. In case of repeated stings, details

Table 2 Mono sensitization and double sensitization detected by intradermal tests and serum specific IgE

\begin{tabular}{lccc}
\hline \multicolumn{4}{c}{ Serum specifc IgE } \\
\hline & $\begin{array}{c}\text { Mono } \\
\text { sensitization }\end{array}$ & $\begin{array}{c}\text { Double } \\
\text { sensitization }\end{array}$ \\
\hline Skin test & Mono sensitization & 284 & 107 \\
& Double sensitization & 84 & 160 \\
\hline
\end{tabular}


on the most severe or - if all stings were tolerated - the most recent field sting were documented: the outcome of 396 field stings (230 of which occurred in double sensitized patients) is summarized in Table 3. 150 field stings took place during VIT, 246 occurred after stopping treatment. Of 396 patients re-stung, 28 reported systemic reactions, implying an overall relapse rate of 7.1\%. During VIT, systemic reactions occurred in 5 stings, and no severe reactions were reported. After discontinuation of VIT, 23 stings led to systemic reactions, 2 of which were classified as severe. Recurring anaphylactic reactions were found to be significantly less severe than the pre-VIT index sting reaction $(P=0.004)$. Characteristics of the 28 patients who suffered systemic anaphylaxis following Hymenoptera field stings during or after VIT are shown in Table 4.

\section{Risk factors for recurring sting-induced anaphylaxis during or after VIT}

Of several putative risk factors or predictors for systemic reactions to Hymenoptera field stings during or after VIT, only one reached statistical significance: severe anaphylaxis following the pre-VIT index sting was clearly related to future recurrence $(P=0.02)$. Tendencies towards an increased risk of relapse in patients reporting repetitive field stings after initiation of VIT $(P=0.07)$ or patients receiving honey bee VIT compared to those receiving Vespula VIT $(P=0.50)$ did not reach statistical significance. Patients with recurring Hymenoptera-venom induced anaphylaxis tended to be older than patients who tolerated field stings, but this was not statistically significant $(P=0.40)$. No relation could be established between recurrent anaphylaxis and the patient's sex $(P=$ $0.70)$, underlying atopy $(P=0.15)$ or asthma $(P=1.0)$, life-time number of Hymenoptera stings $(P=0.46)$, the decline of skin test reactivity $(P=0.45)$ or specific IgE $(P=0.12)$ during VIT, the time interval from index sting to initiation of VIT $(P=0.67)$, and the duration of VIT $(P=0.56)$.

\section{Treatment efficacy in mono sensitized and double sensitized patients}

The overall relapse rates during and after VIT were $6.0 \%$ in mono sensitized and $7.8 \%$ in double sensitized patients. Though there was a tendency towards an increased risk of treatment failure, double sensitization was not significantly related to relapsing sting-induced anaphylaxis $(P=0.56$, Table 5$)$. This tendency, while not reaching statistical relevance, was more pronounced in the above defined subgroup of double sensitized patients with equal reactivity to both venoms in diagnostic tests $(P=0.15)$.

\section{Discussion}

Standard dosed single venom-based immunotherapy over 3-5 years results in effective and long-lasting protection.

VIT constitutes an effective treatment to protect venom allergic patients from sting-induced anaphylaxis [20]. Protection is highest during the maintenance phase and tends to decrease after discontinuation of treatment, resulting in overall relapse rates of approximately 10 to $15 \%$ [15,21-27]. Relapses following VIT-initiation tend to be less severe than pre-VIT sting reactions [23,24,27], but serious and even fatal Hymenoptera venom-induced anaphylaxis does recur on rare occasions [22,28-30]. We herein present the hitherto largest [22-26,31] single centre observational cohort study investigating the longterm outcome of VIT in a group of 635 Hymenoptera venom-allergic patients who received standard dosed treatment for 3 to 5 years between 1988 and 2008 (postVIT observation time was up to 17 years). In contrast to other studies based on either challenge stings [27,32-35] or field-sting-related observations [23-26,31], all patients included received single VIT. Basic epidemiologic

Table 3 Clinical outcome of Hymenoptera field stings during and after VIT

\begin{tabular}{|c|c|c|c|c|c|}
\hline & \multirow[t]{2}{*}{ No reaction } & \multicolumn{3}{|c|}{ Anaphylactic reaction } & \multirow[t]{2}{*}{ Total } \\
\hline & & I (mild) & II (moderate) & III (severe) & \\
\hline All stings & $368(92.9 \%)$ & $13(3.3 \%)$ & $13(3.3 \%)$ & $2(0.5 \%)$ & $396(100 \%)$ \\
\hline Mono sensitized patients & $156(94.0 \%)$ & $6(3.6 \%)$ & $4(2.4 \%)$ & $0(0 \%)$ & $166(100 \%)$ \\
\hline Double sensitized patients & $212(92.2 \%)$ & $7(3.0 \%)$ & $9(3.9 \%)$ & $2(0.9 \%)$ & $230(100 \%)$ \\
\hline Stings during VIT & $145(96.7 \%)$ & $3(2.0 \%)$ & $2(1.3 \%)$ & $0(0 \%)$ & $150(100 \%)$ \\
\hline Mono sensitized patients & $67(98.5 \%)$ & $1(1.5 \%)$ & $0(0 \%)$ & $0(0 \%)$ & $68(100 \%)$ \\
\hline Double sensitized patients & $78(95.1 \%)$ & $2(2.4 \%)$ & $2(2.4 \%)$ & $0(0 \%)$ & $82(100 \%)$ \\
\hline Stings after VIT discontinuation & $223(90.7 \%)$ & $10(4.1 \%)$ & $11(4.5 \%)$ & $2(0.8 \%)$ & $246(100 \%)$ \\
\hline Mono sensitized patients & $89(90.8 \%)$ & $5(5.1 \%)$ & $4(4.1 \%)$ & $0(0 \%)$ & $98(100 \%)$ \\
\hline Double sensitized patients & $134(90.5 \%)$ & $5(3.4 \%)$ & $7(4.7 \%)$ & $2(1.4 \%)$ & $148(100 \%)$ \\
\hline
\end{tabular}


Table 4 Characteristics of 28 patients suffering re-sting reactions during or after VIT

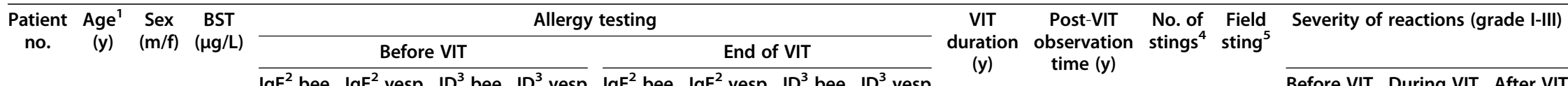
Patients receiving honey bee VIT

$\begin{array}{lllll}1 & 43 & \mathrm{~m} & \text { n.d. } & 3 \\ 2 & 20 & \mathrm{~m} & \text { n.d. } & 6 \\ 3 & 39 & \mathrm{f} & \text { n.d. } & 3 \\ 4 & 53 & \mathrm{f} & \text { n.d. } & 2 \\ 5 & 53 & \mathrm{~m} & \text { n.d. } & 6 \\ 6 & 37 & \mathrm{f} & \text { n.d. } & 3 \\ 7 & 26 & \mathrm{~m} & \text { n.d. } & 3 \\ 8 & 11 & \mathrm{~m} & \text { n.d. } & 3 \\ 9 & 28 & \mathrm{f} & \text { n.d. } & 4\end{array}$

Patients receiving Vespula VIT

\begin{tabular}{|c|c|c|c|c|c|c|c|c|c|c|c|c|c|c|c|c|c|c|}
\hline 10 & 18 & $f$ & n.d. & 0 & 3 & neg & 0.01 & 0 & 2 & neg & 0.01 & 5 & 12 & 2 & vesp & $\|$ & $L R$ & I \\
\hline 11 & 50 & $f$ & 7.63 & 0 & 2 & neg & 0.1 & 0 & 3 & neg & 1 & 5 & 2 & 2 & vesp & $\|$ & $L R$ & $\|$ \\
\hline 12 & 39 & $f$ & n.d. & 0 & 2 & neg & 0.1 & 0 & 0 & neg & 0.01 & 3 & 8 & 6 & vesp & III & - & I \\
\hline 13 & 21 & $\mathrm{~m}$ & 4,97 & 0 & 3 & neg & 0.001 & 0 & 2 & neg & 0.1 & 3 & 8 & 3 & vesp & $\|$ & - & $\|$ \\
\hline 14 & 47 & $f$ & n.d. & 0 & 2 & neg & 0.1 & 0 & 2 & neg & 1 & 5 & 4 & 1 & vesp & III & - & $\|$ \\
\hline 15 & 51 & $\mathrm{~m}$ & n.d. & 0 & 3 & neg & 0.001 & 0 & 0 & neg & 0.1 & 3 & 11 & 1 & vesp & III & - & I \\
\hline 16 & 48 & $f$ & n.d. & 0 & 2 & neg & 0.01 & 0 & 2 & neg & 0.1 & 5 & 4 & 1 & u & 1 & - & ॥ \\
\hline 17 & 30 & $f$ & n.d. & 0 & 3 & neg & 0.1 & 0 & 0 & neg & 1 & 3 & 10 & 1 & u & $\|$ & 1 & - \\
\hline 18 & 56 & $f$ & n.d. & 0 & 5 & neg & 0.001 & 0 & 3 & neg & 0.1 & 5 & 7 & 1 & vesp & $\|$ & - & I \\
\hline 19 & 32 & $\mathrm{~m}$ & n.d. & 1 & 1 & neg & 0.1 & 0 & 0 & 1 & 0.1 & 4 & 13 & 3 & vesp & III & $L R$ & $\|$ \\
\hline 20 & 54 & $\mathrm{~m}$ & 6.69 & 3 & 2 & 1 & 0.1 & 2 & 2 & neg & 0.1 & 3 & 13 & 4 & vesp & III & - & III \\
\hline 21 & 53 & $\mathrm{~m}$ & 110,0 & 3 & 3 & 0.1 & 0.01 & 0 & 1 & 1 & 1 & 3 & 11 & 3 & u & III & - & $\|$ \\
\hline 22 & 45 & $\mathrm{~m}$ & n.d. & 2 & 2 & 1 & 0.01 & 1 & 3 & neg & 0.1 & 5 & 4 & 3 & vesp & III & $\|$ & - \\
\hline 23 & 30 & $\mathrm{~m}$ & n.d. & 3 & 4 & 0.1 & 0.001 & 0 & 1 & 1 & 0.1 & 3 & 9 & 2 & vesp & I & - & III \\
\hline 24 & 55 & $f$ & n.d. & 2 & 2 & 0.01 & 0.01 & 0 & 0 & 1 & 0.1 & 4 & 8 & 2 & vesp & $\|$ & 1 & $L R$ \\
\hline 25 & 40 & $\mathrm{~m}$ & n.d. & 2 & 3 & 0.01 & 0.001 & n.d. & n.d. & n.d. & n.d. & 3 & 14 & 1 & u & III & - & I \\
\hline 26 & 37 & $\mathrm{~m}$ & n.d. & 2 & 3 & 0.01 & 0.01 & 2 & 2 & 0.1 & 1 & 3 & 9 & 3 & u & III & - & $\|$ \\
\hline
\end{tabular}


Table 4 Characteristics of $\mathbf{2 8}$ patients suffering re-sting reactions during or after VIT (Continued)

\begin{tabular}{|c|c|c|c|c|c|c|c|c|c|c|c|c|c|c|c|c|c|c|}
\hline 27 & 39 & $\bar{f}$ & n.d. & 2 & 5 & 1 & 0.001 & 1 & 2 & 1 & 0.1 & 6 & 9 & 2 & vesp & $\|$ & - & $\overline{\|}$ \\
\hline 28 & 46 & $f$ & n.d. & 2 & 2 & neq & n.d. & 2 & 2 & 1 & 0.1 & 3 & 13 & 3 & vesp & 1 & - & $\|$ \\
\hline
\end{tabular}

${ }^{1}$ Age at the time of VIT initiation, ${ }^{2}$ figures refer to semi-quantitative serological classes, ${ }^{3}$ figures refer to intradermal test endpoint concentrations ( $\left.\mu \mathrm{g} / \mathrm{mL}\right),{ }^{4}$ number of Hymenoptera stings after VIT initiation,

Age at the time of VIT initiation, 'figures refer to semi-quantitative serological classes, figures refer to intradermal test endpoint concentrations $(\mu \mathrm{g} / \mathrm{mL})$, number of Hymenoptera stings after VIT initiation
${ }^{5}$ Hymenoptera sting eliciting systemic reaction during or after VIT.

${ }^{5}$ Hymenoptera sting eliciting systemic reaction during or after VIT.
Abbreviations: bee honey bee, BST baseline serum tryptase, ID intradermal skin test, $L R$ local reaction, n.d. not determined, neg negative, vesp, Vespula species, VIT venom immunotherapy, $u$ unknown. 
Table 5 Impact of double sensitization on the outcome of treatment

\begin{tabular}{ccccc}
\hline & $\begin{array}{c}\text { Mono sensitized } \\
\text { patients }\end{array}$ & \multicolumn{2}{c}{$\begin{array}{c}\text { Double sensitized } \\
\text { patients }\end{array}$} & Total cohort \\
\hline Tolerated & $156(94.0 \%)$ & $212(92.2 \%)$ & $73(89.0 \%)$ & $368(92.9 \%)$ \\
field sting & & All & Subgroup* \\
Relapse & $10(6.0 \%)$ & $18(7.8 \%)^{1}$ & $9(11.0 \%)^{2}$ & $28(7.1 \%)$ \\
Sum & $166(100 \%)$ & $230(100 \%)$ & $82(100 \%)$ & $396(100 \%)$ \\
\hline
\end{tabular}

*Subgroup of double sensitized patients comprising individuals with equal reactivity to both venoms in diagnostic tests.

${ }^{1} P=0.56$ ( $P$-values result from statistical comparison of the risk of relapse in mono sensitized and double sensitized patients).

${ }^{2} P=0.15$.

findings such as a median age of 39 years at the time of VIT initiation, a slight male preponderance being more pronounced in bee venom allergic patients [36], and a $21.3 \%$ rate of severe anaphylactic reactions following pre-VIT index stings as well as the re-exposure rate of $62.4 \%$ (to any Hymenoptera sting after VIT-initiation) and the overall relapse rate of $7.1 \%$ (that is $3.3 \%$ during VIT and $9.3 \%$ after its discontinuation) are roughly in line with those reported in earlier studies. Recurrent anaphylaxis to Hymenoptera field stings was significantly less severe than the pre-VIT index sting reaction $(P=$ 0.004 ) which is again in accordance with previous findings [24,27]. Grade III anaphylaxis recurred in only 2 individuals (Table 4, patients 20 and 23) following discontinuation of treatment (that is $0.5 \%$ of 396 patients suffering re-stings). Number 23 was the only patient to develop more severe anaphylaxis on the occasion of his post-VIT field sting than before VIT. He got re-stung 6 years after discontinuation of treatment by more than 20 yellow jackets emerging from their nest. He did not use his epinephrine injector and lost consciousness shortly after developing generalized wheals.

We conclude that standard dosed single venom-based immunotherapy over 3 to 5 years effectively and longlastingly protects the vast majority of Hymenoptera venom allergic patients.

\section{Treatment failure is related to a limited number of risk factors}

Of a number of potential risk factors for treatment failure, only one reached statistical significance: severe anaphylaxis following the pre-VIT index sting was indicative of a higher risk of recurrence $(P=0.02)$ which is in accordance with the results of some previous studies [31,34], but was not supported by others [24,26,27,32,37]. Case series and epidemiological studies have clearly documented that both, the severity of anaphylaxis to the index sting as well as the risk of treatment failure, are related to an increase of the baseline serum tryptase concentration [38-41]. Tryptase determination is nowadays an integrative part of diagnostic assessment in Hymenoptera venom allergic patients, but was not routinely available during the first decade of our study. As a result, we could not retrospectively correlate the risk of treatment failure with baseline serum tryptase concentrations in our study group (tryptase concentrations were available in 87 patients and exceeding $11.4 \mu \mathrm{g} / \mathrm{L}$ in 9 cases).

Other potential risk factors including the patient's age $(P=0.40)$, sex $(P=0.70)$, and preexisting atopy $(P=0.15)$, as well as the decline of venom specific $\operatorname{IgE}(P=0.12)$ and skin test reactivity $(P=0.45)$ during VIT $[24,25,27,31,42]$, the duration of treatment [24,34] $(P=0.56)$, the culprit insect [36] $(P=0.50)$, and repetitive exposure to Hymenoptera field stings $(P=0.07)$ did not significantly correlate with the outcome of VIT in our patient cohort. At least two aspects, however, require critical reflection: i) Higher relapse rates have been observed in honey bee venom allergic patients by other authors [36]. We assume that our inability to confirm a relation between the culprit stinging insect and the outcome of VIT may be due to a statistical type 2 error, meaning that the association would have proved statistically significant in a cohort of patients containing a higher proportion of honey bee venom allergic individuals. ii) Though there was a tendency towards an increased risk of relapse in individuals repeatedly suffering Hymenoptera re-stings during and after VIT $(P=$ 0.07 ), we could not statistically confirm repetitive stings as a risk factor for treatment failure as reported in previous studies $[23,24]$. Our findings, however, demonstrate that tolerated field stings do not reliably predict long-term protection as illustrated by patients $2,3,10,11$, and 19 (Table 4) who did not react to Hymenoptera stings during VIT, but had anaphylactic reactions to re-stings following discontinuation of treatment.

\section{Most double sensitized patients are sufficiently protected by single VIT}

In Northern Europe, the vast majority of sting-related anaphylactic reactions can be attributed to stings of honey bees and Vespula species, Vespula stings being the most common elicitor of venom-induced anaphylaxis in Germany. Our 55.3\% rate of patients with double sensitization to both venoms is in line with previous studies reporting up to $59 \%$ of double positive sera [4], depending on the system used for determination of specific IgE $[4,43]$. Double positivity was more often detected serologically than in intradermal tests (Table 2) which is again in accordance with earlier findings [43]. As described previously [4,36], serological $(P<0.001)$ and intradermal test reactivity $(P=0.001)$ as well as the rate of double positivity $(P<0.001)$ were higher in bee venom allergic patients.

Other than venom "cross-reactivity" due to partial sequence identity of protein allergens or cross-reactive 
carbohydrate determinants, "independent" or major allergen-based double sensitization, has been claimed to necessitate VIT with both venoms $[4,6,10,13]$. A historical study published in 1992, however, suggested that single venom-based immunotherapy effectively protects most patients despite reactivity to more than one venom [44], but did not statistically compare the outcome of VIT in mono sensitized and double sensitized individuals.

In our allergy clinic, VIT is usually restricted to a single venom (see Methods section and Figure 1 for the process of decision making). During the study period, only 14 patients received double VIT with honey bee and Vespula venom and were therefore excluded from evaluation (no relapses were reported in this small group of patients). Our approach is based on the assumption that sensitization to one or several Hymenoptera venoms is a common finding in approximately $25 \%$ of an average adult population $[15,45]$, reflecting previous exposure to Hymenoptera stings rather than clinical allergy. Though asymptomatic sensitization is associated with an intermediate risk of subsequent sting-related anaphylaxis (which may reach $17 \%[15,46]$ ), it is international consensus that it is not an indication for VIT. In our opinion this also applies to patients with a history of anaphylactic sting reactions to one insect and a hitherto asymptomatic sensitization to another - regardless of its pattern or origin.

Our study specifically compared the efficacy of single venom-based immunotherapy in mono sensitized and double sensitized individuals. Relapse rates in both groups $(6.0 \%$ in mono sensitized and $7.8 \%$ in double sensitized patients) were within or even below commonly reported recurrence rates of 10 to $15 \%$. Still, there was a tendency (though not statistically significant) towards an increased risk of field sting-related anaphylaxis in double sensitized patients $(P=0.56)$. Of 28 patients suffering re-sting reactions, 5 double sensitized individuals reacted to an unidentified stinging insect (Table 4, patients 7, 8, 21, 25, and 26). One double sensitized patient (patient 5) treated with honey bee VIT subsequently reacted to a Vespula sting with a grade I reaction. This indicates a remaining risk of a wrong or insufficient choice of venom when using our above mentioned algorithm which is based on the patient's ability to provide correct stingrelated information or even visual identification of the culprit stinging insect. Study data, however, demonstrate that the future risk of anaphylactic reactions to either honey bee or yellow jacket stings was well below the reported $17 \%$ rate $[15,46]$ of anaphylactic sting reactions in healthy adults with asymptomatic sensitization. While still not reaching statistical significance, there was a tendency towards an increased risk of relapsing sting-induced anaphylaxis in patients with equal reactivity to both venoms in diagnostic tests $(P=0.15)$ when compared to the total group of double sensitized patients $(P=0.56)$. We conclude that, in double sensitized individuals, the respective degree of skin test and serological reactivity to both venoms is indicative of their clinical relevance, meaning that the culprit insect venom is likely to produce a stronger response in diagnostic tests. It is self-evident that, since there is no absolute correlation between specific IgE levels or the degree of skin test reactivity with clinical responses to Hymenoptera stings [1], this conclusion is restricted to the intra-individual comparison of test results in double sensitized patients.

The overall low rate of relapsing anaphylaxis following Hymenoptera field stings (7.1\%) and the exceedingly low rate of severe allergic reactions $(0.5 \%)$ as observed in our patient cohort confirm effective long-term protection of mono sensitized and most double sensitized individuals by single VIT. We conclude that time-consuming and expensive double VIT should reasonably be reserved to clinically double allergic patients with a history of systemic reactions to both venoms. It may additionally be considered in double sensitive patients with equal reactivity to both venoms who have not reliably identified the responsible stinging insect. In cases of doubt, additional risk factors such as an elevated baseline serum tryptase, a history of particularly severe anaphylactic reactions to pre-VIT index stings, or a high degree of exposure to Hymenoptera stings should be taken into account. The recent introduction of assays determining specific IgE to recombinant major allergens rApi $\mathrm{m} 1$, rVes v5 and rVes v1 [4-6] might further help to identify double sensitized individuals at an increased risk of future anaphylactic reactions to their "non-culprit" insect. However, prospective clinical trials are needed to investigate the positive predictive value of major allergen-based IgE-sensitization.

\section{Conclusions and clinical implications}

i) In addition to a comprehensive history, the respective degree of skin test and serological reactivity should be taken into account to identify the appropriate venom for immunotherapy in double sensitized patients.

ii) 3 to 5 years of standard-dosed single venom-based immunotherapy result in long-term effective protection of the vast majority of Hymenoptera venom allergic patients. Recurring anaphylaxis to Hymenoptera field-stings during or after VIT is generally less severe than the pre-VIT sting reaction.

iii) Treatment failure is related to a limited number of risk factors including a history of severe pre-VIT sting-induced anaphylaxis.

iv) No significant correlation between double sensitization and relapsing venom-induced 
anaphylaxis could be statistically confirmed in our study group, indicating that VIT can be reasonably confined to a single venom in most patients.

\section{Abbreviation \\ VIT: (Venom immunotherapy).}

\section{Competing interests}

The authors declare that they have no competing interest.

\section{Authors' contribution}

JS participated in the design of the study, collection and interpretation of data, and drafted the manuscript. BH participated in the design of the study, collected the data, and assisted in drafting the manuscript. $J \mathrm{H}$ performed the statistical analysis, and helped to draft the manuscript. AK participated in the collection of data, and critically revised the manuscript. AT participated in the design of the study, collection and interpretation of data, and critically revised the manuscript. All authors read and approved the final manuscript.

\section{Acknowledgements}

This publication was funded by the German Research Foundation (DFG) and the University of Würzburg in the funding programme Open Access Publishing.

\section{Author details}

'Department of Dermatology and Allergy, University Hospital Würzburg, Würzburg, Germany. ${ }^{2}$ Department of Medicine, University Hospital of Mannheim, Mannheim, Germany. Institute of Mathematics, Department of Statistics, University of Würzburg, Würzburg, Germany.

Received: 27 April 2013 Accepted: 10 August 2013

Published: 2 September 2013

\section{References}

1. Golden DB, Moffitt J, Nicklas RA, Freeman T, Graft DF, Reisman RE, Tracy JM, Bernstein D, Blessing-Moore J, Cox L: Stinging insect hypersensitivity: a practice parameter update 2011. J Allergy Clin Immunol 2011, 127:852-854. e851-823.

2. Krishna MT, Ewan PW, Diwakar L, Durham SR, Frew AJ, Leech SC, Nasser SM: Diagnosis and management of hymenoptera venom allergy: British Society for Allergy and Clinical Immunology (BSACl) guidelines. Clin Exp Allergy 2011, 41:1201-1220.

3. Bonifazi F, Jutel M, Bilò BM, Birnbaum J, Müller U: Prevention and treatment of hymenoptera venom allergy: guidelines for clinical practice. Allergy 2005, 60:1459-1470.

4. Müller UR, Johansen N, Petersen AB, Fromberg-Nielsen J, Haeberli G: Hymenoptera venom allergy: analysis of double positivity to honey bee and Vespula venom by estimation of IgE antibodies to species-specific major allergens Api $\mathrm{m} 1$ and Ves v5. Allergy 2009, 64:543-548.

5. Müller U, Schmid-Grendelmeier P, Hausmann O, Helbling A: IgE to recombinant allergens Api $m 1$, Ves $v 1$, and Ves $v 5$ distinguish double sensitization from crossreaction in venom allergy. Allergy 2012. 67:1069-1073.

6. Hofmann SC, Pfender N, Weckesser S, Huss-Marp J, Jakob T: Added value of IgE detection to rApi $\mathrm{m} 1$ and rVes $v 5$ in patients with Hymenoptera venom allergy. J Allergy Clin Immunol 2011, 127:265-267.

7. Mittermann I, Zidarn M, Silar M, Markovic-Housley Z, Aberer W, Korosec P, Kosnik M, Valenta R: Recombinant allergen-based lgE testing to distinguish bee and wasp allergy. J Allergy Clin Immunol 2010, 125:1300-1307. e1303.

8. Jappe U, Raulf-Heimsoth M, Hoffmann M, Burow G, Hübsch-Müller C, Enk A: In vitro hymenoptera venom allergy diagnosis: improved by screening for cross-reactive carbohydrate determinants and reciprocal inhibition. Allergy 2006, 61:1220-1229.

9. Hemmer W, Focke M, Kolarich D, Wilson IB, Altmann F, Wohrl S, Gotz M, Jarisch R: Antibody binding to venom carbohydrates is a frequent cause for double positivity to honeybee and yellow jacket venom in patients with stinging-insect allergy. J Allergy Clin Immunol 2001, 108:1045-1052.

10. Straumann F, Bucher C, Wüthrich B: Double sensitization to honeybee and wasp venom: immunotherapy with one or with both venoms? Value of
FEIA inhibition for the identification of the cross-reacting ige antibodies in double-sensitized patients to honeybee and wasp venom. Int Arch Allergy Immunol 2000, 123:268-274.

11. Reisman RE, Müller UR, Wypych Jl, Lazell Ml: Studies of coexisting honeybee and vespid-venom sensitivity. J Allergy Clin Immunol 1984, 73:246-252.

12. Sturm GJ, Böhm E, Trummer M, Weiglhofer I, Heinemann A, Aberer W: The CD63 basophil activation test in Hymenoptera venom allergy: a prospective study. Allergy 2004, 59:1110-1117.

13. Eberlein B, Krischan L, Darsow U, Ollert M, Ring J: Double positivity to bee and wasp venom: improved diagnostic procedure by recombinant allergen-based IgE testing and basophil activation test including data about cross-reactive carbohydrate determinants. J Allergy Clin Immunol 2012, 130:155-161.

14. Mertens M, Amler S, Moerschbacher BM, Brehler R: Cross-reactive carbohydrate determinants strongly affect the results of the basophil activation test in hymenoptera-venom allergy. Clin Exp Allergy 2010, 40:1333-1345.

15. Golden DB: Insect sting allergy and venom immunotherapy: a model and a mystery. J Allergy Clin Immunol 2005, 115:439-447.

16. Muraro A, Roberts G, Clark A, Eigenmann PA, Halken S, Lack G, MoneretVautrin A, Niggemann B, Rance F: The management of anaphylaxis in childhood: position paper of the European academy of allergology and clinical immunology. Allergy 2007, 62:857-871.

17. Stoevesandt J, Hain J, Kerstan A, Trautmann A: Over- and underestimated parameters in severe Hymenoptera venom-induced anaphylaxis: cardiovascular medication and absence of urticaria/angioedema. J Allergy Clin Immunol 2012, 130:698-704.

18. Position paper: Allergen standardization and skin tests. The European Academy of Allergology and Clinical Immunology. Allergy 1993, 48:48-82.

19. Bilò BM, Ruëff F, Mosbech $H$, Bonifazi F, Oude-Elberink JN: Diagnosis of Hymenoptera venom allergy. Allergy 2005, 60:1339-1349.

20. Boyle RJ, Elremeli M, Hockenhull J, Cherry MG, Bulsara MK, Daniels M, Oude Elberink JN: Venom immunotherapy for preventing allergic reactions to insect stings. Cochrane Database Syst Rev 2012, 10:008838.

21. Golden DB: Long-term outcome after venom immunotherapy. Curr Opin Allergy Clin Immunol 2010, 10:337-341.

22. Golden DB, Kagey-Sobotka A, Lichtenstein LM: Survey of patients after discontinuing venom immunotherapy. J Allergy Clin Immunol 2000, 105:385-390.

23. Hafner T, DuBuske L, Kosnik M: Long-term efficacy of venom immunotherapy. Ann Allergy Asthma Immunol 2008, 100:162-165.

24. Lerch E, Müller UR: Long-term protection after stopping venom immunotherapy: results of re-stings in 200 patients. J Allergy Clin Immunol 1998, 101:606-612.

25. Reisman RE, Lantner R: Further observations of stopping venom immunotherapy: comparison of patients stopped because of a fall in serum venom-specific lgE to insignificant levels with patients stopped prematurely by self-choice. J Allergy Clin Immunol 1989, 83:1049-1054.

26. Golden DB, Kwiterovich KA, Kagey-Sobotka A, Lichtenstein LM: Discontinuing venom immunotherapy: extended observations. J Allergy Clin Immunol 1998, 101:298-305.

27. Golden DB, Kwiterovich KA, Kagey-Sobotka A, Valentine MD, Lichtenstein LM: Discontinuing venom immunotherapy: outcome after five years. J Allergy Clin Immunol 1996, 97:579-587.

28. Oude Elberink JN, de Monchy JG, Kors JW, van Doormaal JJ, Dubois AE: Fatal anaphylaxis after a yellow jacket sting, despite venom immunotherapy, in two patients with mastocytosis. J Allergy Clin Immunol 1997, 99:153-154.

29. Light WC: Insect sting fatality 9 years after venom treatment (venom allergy, fatality). J Allergy Clin Immunol 2001, 107:925.

30. Golden DB: Fatal insect allergy after discontinuation of venom immunotherapy. J Allergy Clin Immunol 2001, 107:925-926.

31. Reisman RE: Duration of venom immunotherapy: relationship to the severity of symptoms of initial insect sting anaphylaxis. J Allergy Clin Immunol 1993, 92:831-836.

32. Müller U, Berchtold $E$, Helbling A: Honeybee venom allergy: results of a sting challenge 1 year after stopping successful venom immunotherapy in 86 patients. J Allergy Clin Immunol 1991, 87:702-709.

33. Haugaard L, Norregaard OF, Dahl R: In-hospital sting challenge in insect venom-allergic patients after stopping venom immunotherapy. J Allergy Clin Immunol 1991, 87:699-702. 
34. Keating MU, Kagey-Sobotka A, Hamilton RG, Yunginger JW: Clinical and immunologic follow-up of patients who stop venom immunotherapy. J Allergy Clin Immunol 1991, 88:339-348.

35. van Halteren HK, van der Linden PW, Burgers JA, Bartelink AK:

Discontinuation of yellow jacket venom immunotherapy: follow-up of 75 patients by means of deliberate sting challenge. J Allergy Clin Immunol 1997, 100:767-770.

36. Müller U, Helbling A, Berchtold E: Immunotherapy with honeybee venom and yellow jacket venom is different regarding efficacy and safety. J Allergy Clin Immunol 1992, 89:529-535.

37. Golden DB, Kagey-Sobotka A, Valentine MD, Lichtenstein LM: Dose dependence of Hymenoptera venom immunotherapy. I Allergy Clin Immunol 1981, 67:370-374.

38. Haeberli G, Bronnimann M, Hunziker T, Müller U: Elevated basal serum tryptase and hymenoptera venom allergy: relation to severity of sting reactions and to safety and efficacy of venom immunotherapy. Clin Exp Allergy 2003, 33:1216-1220.

39. Ruëff F, Przybilla B, Bilò MB, Müller U, Scheipl F, Aberer W, Birnbaum J, Bodzenta-Lukaszyk A, Bonifazi F, Bucher C, et al: Predictors of severe systemic anaphylactic reactions in patients with Hymenoptera venom allergy: importance of baseline serum tryptase-a study of the European Academy of Allergology and Clinical Immunology Interest Group on Insect Venom Hypersensitivity. J Allergy Clin Immunol 2009, 124:1047-1054.

40. Niedoszytko M, de Monchy J, van Doormaal JJ, Jassem E, Oude Elberink JN Mastocytosis and insect venom allergy: diagnosis, safety and efficacy of venom immunotherapy. Allergy 2009, 64:1237-1245.

41. González De Olano D, Alvarez-Twose I, Esteban-López MI, Sánchez-Muñoz L, de Durana MD, Vega A, García-Montero A, González-Mancebo E, Belver T, Herrero-Gil MD: Safety and effectiveness of immunotherapy in patients with indolent systemic mastocytosis presenting with Hymenoptera venom anaphylaxis. J Allergy Clin Immunol 2008, 121:519-526.

42. Randolph CC, Reisman RE: Evaluation of decline in serum venom-specific IgE as a criterion for stopping venom immunotherapy. J Allergy Clin Immunol 1986, 77:823-827.

43. Egner W, Ward C, Brown DL, Ewan PW: The frequency and clinical significance of specific lgE to both wasp (Vespula) and honey-bee (Apis) venoms in the same patient. Clin Exp Allergy 1998, 28:26-34.

44. Reisman RE, Livingston A: Venom immunotherapy: 10 years of experience with administration of single venoms and 50 micrograms maintenance doses. J Allergy Clin Immunol 1992, 89:1189-1195.

45. Golden DB, Marsh DG, Kagey-Sobotka A, Freidhoff L, Szklo M, Valentine MD, Lichtenstein LM: Epidemiology of insect venom sensitivity. JAMA 1989, 262:240-244.

46. Golden DB, Marsh DG, Freidhoff LR, Kwiterovich KA, Addison B, KageySobotka A, Lichtenstein LM: Natural history of Hymenoptera venom sensitivity in adults. J Allergy Clin Immunol 1997, 100:760-766.

doi:10.1186/1710-1492-9-33

Cite this article as: Stoevesandt et al.: Single venom-based immunotherapy effectively protects patients with double positive tests to honey bee and Vespula venom. Allergy, Asthma \& Clinical Immunology 2013 9:33.

\section{Submit your next manuscript to BioMed Central and take full advantage of:}

- Convenient online submission

- Thorough peer review

- No space constraints or color figure charges

- Immediate publication on acceptance

- Inclusion in PubMed, CAS, Scopus and Google Scholar

- Research which is freely available for redistribution 